\title{
Posterior approach for anteriorly located cervical spinal cord hemangioblastomas: technical note
}

\author{
Jorn Van Der Veken, MD, ${ }^{1}$ Sven Gläsker, MD, PhD, ${ }^{1}$ Vassilis Vougioukas, MD, $\mathrm{PhD},{ }^{2}$ and \\ Vera Van Velthoven, MD, PhD'

\begin{abstract}
${ }^{1}$ Neurosurgery Department, Universitair Ziekenhuis Brussel, Brussels, Belgium; and ${ }^{2}$ Neurosurgery Department, Metropolitan Hospital Athens, Greece
\end{abstract}

\begin{abstract}
The surgical management of anteriorly located spinal cord hemangioblastomas remains a challenge. Different approaches have been published, of which the anterior approach seems to be the most obvious and commonly used. A posterior approach might be more suitable in certain patients, especially in cases of cystic hemangioblastomas. The authors present 3 cases of anterior spinal hemangioblastomas, which were all resected via a posterior approach. The authors discuss the rationale for choosing this approach and explain the technique in detail.
\end{abstract}

https://thejns.org/doi/abs/10.3171/2018.1.SPINE171108

KEYWORDS hemangioblastoma; intramedullary; surgical technique; cervical; oncology

$\mathrm{S}$ PINAL cord hemangioblastomas are highly vascularized benign tumors, accounting for $2 \%-6 \%$ of all spinal cord tumors. ${ }^{6}$ They are diagnosed either as a solitary lesion (70\%-80\%) or in the setting of von Hippel-Lindau (VHL) disease (20\%-30\%). ${ }^{7}$ Although they are regarded as benign lesions (WHO grade I), due to their localization, highly vascularized nature, and frequent associated edema or syrinx, diagnosis often precedes significant neurological morbidity or even mortality. ${ }^{3}$

Spinal hemangioblastomas are predominantly located in the posterolateral aspect of the spinal cord (98\%) arising from the dorsal root entry zone or dorsal column tracts. ${ }^{2}$ They can be further classified into entirely intramedullary or combined intraextramedullary; only rarely do they grow entirely extramedullary. ${ }^{9}$ The hemangioblastomas developing in the anterior aspect of the spinal cord (anterior of the dentate ligament) mostly grow entirely intramedullary. ${ }^{4}$

Studies on the treatment of these lesions with the use of radiosurgery have been published with differing results, but the gold standard remains microsurgical resection. ${ }^{8}$ The goal of surgery is a total resection without causing a new neurological deficit. Despite all the advances in the neurosurgical armamentarium, this remains challenging when operating in the anterior spinal cord. Due to its rarity, the best surgical strategy when dealing with an anteriorly located hemangioblastoma remains unclear. ${ }^{10}$ Different approaches have been published, of which the anterior approach seems the most obvious and commonly used. A posterior approach might be more suitable in certain patients, especially in cases of cystic hemangioblastomas.

We discuss 3 patients with VHL disease who had an anteriorly located cervical hemangioblastoma, which were all operated on via a posterior approach.

\section{Methods}

All 3 patients were operated on by the senior author (V.V.V.), who has a vast experience with hemangioblastoma surgery. ${ }^{11}$ The functional scale proposed by McCormick and modified by Constantini et al. (Table 1) was used to evaluate the patients' neurological status before and after surgical treatment. ${ }^{1}$

\section{Surgical Technique}

Methylprednisolone is started 24 hours prior to surgery. 
TABLE 1. Modified McCormick clinical-functional scale*

\begin{tabular}{cc}
$\begin{array}{c}\text { Functional } \\
\text { Grade }\end{array}$ & \multicolumn{1}{c}{ Description } \\
\hline I & $\begin{array}{c}\text { Neurologically intact; walks normally; may have minimal } \\
\text { dysesthesia }\end{array}$ \\
\hline II & $\begin{array}{c}\text { Mild motor or sensory deficit; maintains functional } \\
\text { independence (walking, feeding, \& using the bathroom } \\
\text { without external aid) }\end{array}$ \\
\hline III & $\begin{array}{c}\text { Moderate deficit; limitation of function; independent with } \\
\text { external aid }\end{array}$ \\
\hline IV & $\begin{array}{c}\text { More severe motor or sensory deficit; limited function } \\
\text { with dependency }\end{array}$ \\
\hline V & $\begin{array}{c}\text { Paraplegia or quadriplegia (even if there is flickering } \\
\text { movement) }\end{array}$ \\
\hline
\end{tabular}

* Modified with permission from Constantini et al: J Neurosurg 85:1036-1043, 1996 (which was a modification of the original published by McCormick et al. in J Neurosurg 72:523-532, 1990).

Patients are placed in a prone position, freeing the abdomen and thorax from all pressure. Neuromonitoring electrodes are placed (somatosensory evoked potential, motor evoked potential [MEP], and D-wave). The spinal level is determined using fluoroscopy. At the beginning of the surgery an extra bolus of methylprednisolone is given. No preoperative embolization was performed in these cases.

A small midline incision is made centered over the level of the lesion, a minimally invasive retractor is placed (Medtronic quadrant), and a unilateral hemilaminectomy is made. In the patient in case 3 a classic laminectomy was performed. Before opening the dura mater, the location of the pseudocyst and hemangioblastoma is determined using ultrasound and color Doppler modalities. When the correct location is confirmed, the epidural D-wave neuromonitoring catheter is put in place.

The dura is opened under microscope and multiple stacking sutures are placed. Opening of the arachnoid and the midline of the spinal cord is determined. A midline myelotomy is performed in the dorsal median raphe to enter the pseudocyst, and multiple pial stacking sutures are placed to obtain an open corridor. Once the cyst is drained, the ventral hemangioblastoma can be localized visually as a reddish eminence in the cyst wall.
Once the hemangioblastoma is visualized, we attain a surgical situation similar to that for a classic posterior hemangioblastoma. The surface of the tumor is dissected in a circumferential way, during which all the arterial feeders are identified and isolated from the anterior spinal artery to be coagulated with great care and precision, and then to be cut with microscissors. During this part of the procedure, great care is taken to stay in the plane between neural tissue and tumor, because entering the tumor will provoke profuse bleeding and entering neural tissue will produce evident deficits. When all arterial feeders are coagulated and the tumor loses its turgor, the draining vein can be coagulated. The tumor is subsequently removed en bloc. The ventral arachnoid remains intact. The pseudocyst is left in place because spontaneous regression is to be expected after hemangioblastoma removal.

After tumor resection and careful hemostasis, the posterior pia at the level of the myelotomy, the arachnoid, the dura mater, and superficial layers are closed in separate layers.

\section{Results \\ Case 1}

A 26-year-old woman with VHL disease had a history of prior resection of multiple spinal hemangioblastomas at the levels T4-8 and T10. She presented with a worsening gait ataxia, as well as increasing sensory deficits: neuropathic pain and hyperesthesia in the 4 limbs (McCormick grade III).

The initial MRI showed a small, ventral, completely intramedullary hemangioblastoma at the level of C6. Sequential MR images showed the development of a pseudocyst with an increasing myelopathy (Fig. 1A and B). Because of increasing symptoms and the progression of the pseudocyst with myelopathy, surgery was performed.

Via a posterior, transcystic approach the hemangioblastoma could be totally removed. Perioperative neuromonitoring showed a slight decrease of amplitude in the MEPs of the right leg, with a normal D-wave. Immediately postoperatively, there was a mild weakness in the left leg (4/5), which was not present preoperatively. However, this recovered totally over the following weeks (McCormick grade II).

An MRI session performed 6 months after surgery
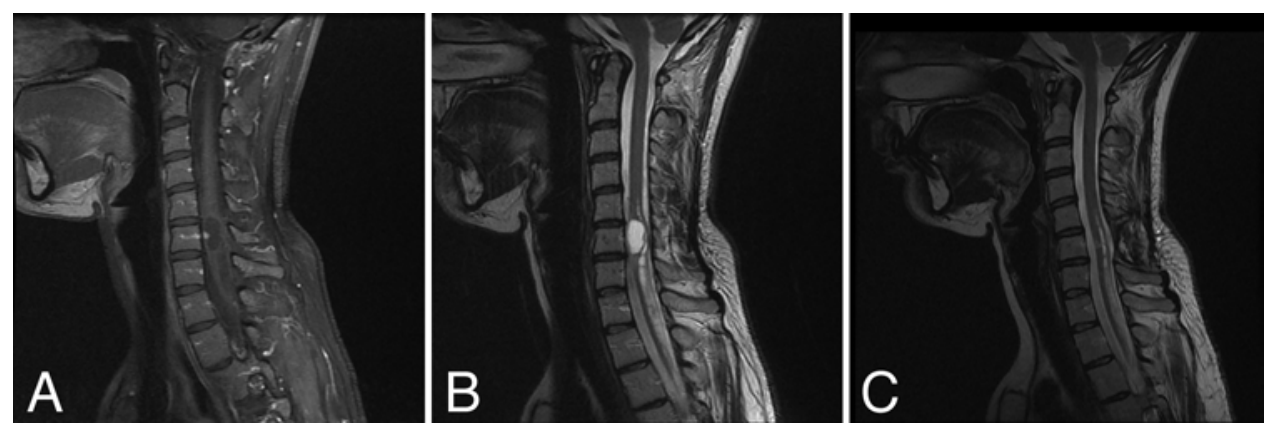

FIG. 1. Case 1. Sagittal T1-weighted MRI study obtained with gadolinium contrast (A) and sagittal T2-weighted MRI study (B) showing an anterior hemangioblastoma with associated extratumoral pseudocyst. Sagittal T2-weighted MRI study (C) obtained 6 months postoperatively showing no residual tumor and disappearing pseudocyst. 


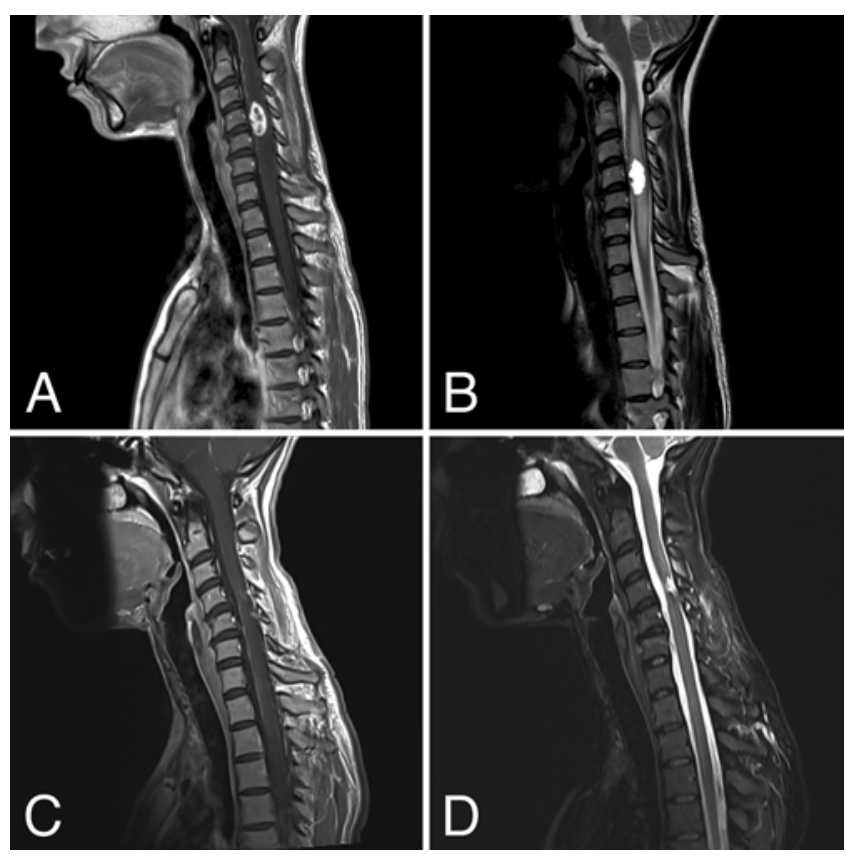

FIG. 2. Case 2. Preoperative sagittal T1-weighted MRI study obtained with gadolinium contrast (A) and T2-weighted MRI study (B) showing an intratumoral cyst. Sagittal T1-weighted MRI study obtained with gadolinium contrast (C) 4 months postoperatively and sagittal T2-weighted MRI study (D) showing a complete resection with small residual cyst.

showed no residual hemangioblastoma and disappearing pseudocyst (Fig. 1C). At 18 months of follow-up there are still neuropathic complaints in the 4 limbs, but there is an improvement of the gait ataxia (McCormick grade II). The MRI findings at 18 months postoperatively were unchanged.

\section{Case 2}

A 35-year-old woman with VHL disease presented with multiple intramedullary hemangioblastomas. Yearly follow-up MRI sessions showed a growing ventral intramedullary lesion at $\mathrm{C} 4-5$ with an increasing perilesional edema. The tumor had a cystic intratumoral component, but no accompanying extratumoral cyst (Fig. 2A and B). The patient had no symptoms. Neurological examination was performed and no peculiarities were found (McCormick grade I).

Because of the significant tumoral growth it was decided to perform surgery. Via a posterior approach the hemangioblastoma could be totally removed. Perioperatively there was a loss of MEPs in the right hemicorpus, but the D-wave remained intact.

Postoperatively the patient developed a right high-grade hemiparesis (McCormick grade IV), which recovered subtotally after an intensive 3-month rehabilitation (McCormick grade II). The 6-month postoperative MRI study showed no residual tumor (Fig. 2C and D). At 12 months there is still a slight motor deficit, but the patient is fully autonomically intact.

\section{Case 3}

A 52-year-old man with VHL disease had mild but increasing weakness in both legs and paresthesias in both hands. Walking was becoming more and more ataxic. Neurological examination showed pyramidal signs, with positive Hofmann-Trömner, bilateral Babinski, and a positive Lhermitte sign (McCormick grade III).

Cervical MRI showed an anteriorly located spinal cord hemangioblastoma, with a large associated pseudocyst and pericystic edema (Fig. 3A and B). Because of the clinical and radiological deterioration, surgery was performed. Via a posterior transcystic approach a complete resection was obtained. Neuromonitoring results remained unchanged during the whole operation.

Postoperatively there was an improvement of the motor deficit and gait abnormalities, and the patient became independent again after 6 weeks of rehabilitation. The postoperative MRI sequence showed no residual tumor (Fig. $3 \mathrm{C}$ ), and 24 months postoperatively the patient is still autonomically intact, with a stable neurological situation.

\section{Discussion}

The anterior location is the most important predictive factor for worse postoperative outcome in ventrally located intramedullary hemangioblastomas. Despite all advances in modern neurosurgery, they remain a challenging surgical venture. ${ }^{4}$

Due to its rarity, few articles on this topic have been published and not all variables are discussed or taken into
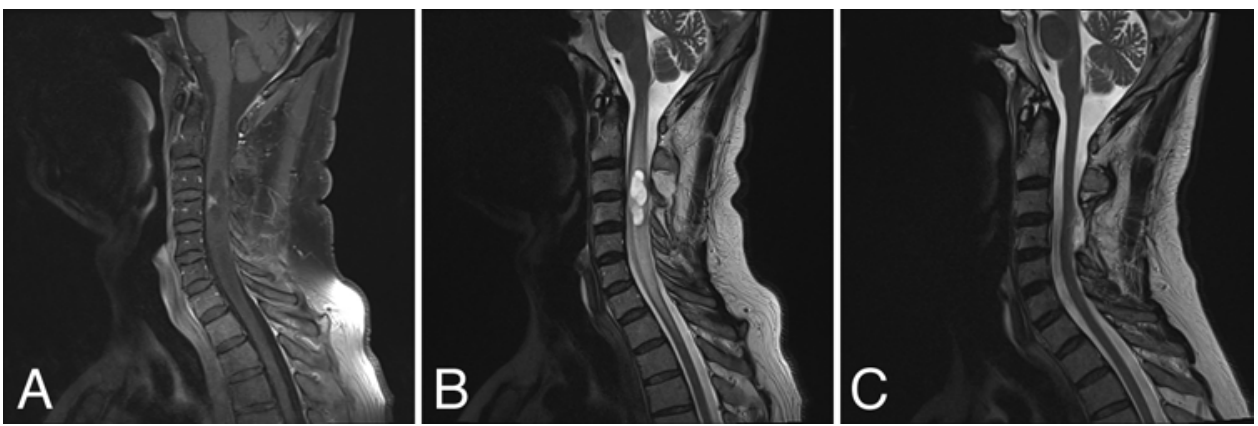

FIG. 3. Case 3. Sagittal T1-weighted MRI study obtained with gadolinium contrast (A) and sagittal T2-weighted MRI study (B) showing an anterior hemangioblastoma with associated extratumoral pseudocyst. Sagittal T2-weighted MRI study (C) obtained 24 months postoperatively showing no residual pseudocyst. 
account when dealing with an anterior intramedullary hemangioblastoma. When the determination for surgery is made, the best approach should be discussed, tailored to the individual radiological characteristics of each hemangioblastoma.

In 2003 a comparison between an anterior and a posterior approach in treating spinal hemangioblastomas was published, with 5 lesions treated via a posterior and 3 via an anterior approach. Although the small patient group was insufficient to perform a statistical comparison, the anterior approach was regarded as superior. ${ }^{9}$

Logically, one would approach an anterior lesion via an anterior approach-however, there are some drawbacks. First, the ventral hemangioblastomas are rarely completely superficial, necessitating an anterior myelotomy or manipulation of the anterior spinal cord, which both increase the risk of postoperative motor deficit. Second, the arterial supply of the anterior two-thirds of the spinal cord is delivered via a single anterior spinal artery, whereas the posterior third is supplied via paired posterior arteries; hence the risk of causing spinal cord ischemia is increased via an anterior approach. ${ }^{5}$ And third, an invasive corpectomy is mandatory in the anterior approach, which increases perioperative morbidity and necessitates the placement of interbody fusion material. The latter complicates the radiological follow-up, due to the important artifacts. Furthermore, the presence of an arthrodesis might also complicate further reinterventions because multiple tumors might continue to occur during the evolution of VHL disease. These obstacles are avoided when using the posterior approach.

In the situation of a superficial ventral hemangioblastoma without associated pseudocyst, where surgery would be indicated, an anterior approach is recommended.

\section{Conclusions}

We want to emphasize that we do not advocate the posterior approach for every anterior spinal hemangioblastoma. However, in the typical presentation in which a large associated cyst has created a working corridor to the tumor, this approach can be used to avoid the inherent risks of the anterior approach. Without a facilitating extratumoral cyst, however, choosing the optimal approach remains very challenging, and waiting for the development of an extratumoral pseudocyst might be the preferred option.

More publications and larger case series are needed in order to tailor the best surgical treatment for anterior spinal cord hemangioblastomas.

\section{References}

1. Constantini S, Houten J, Miller DC, Freed D, Ozek MM, Rorke LB, et al: Intramedullary spinal cord tumors in chil- dren under the age of 3 years. J Neurosurg 85:1036-1043, 1996

2. Hurth M, André J, Djindjian R, Escourolle R, Houdart R, Poirier J, et al: Les hémangioblastomes intrarachidiens. Neurochirurgie 21 (Suppl 1):1-136, 1975

3. Lonser RR, Oldfield EH: Spinal cord hemangioblastomas. Neurosurg Clin N Am 17:37-44, 2006

4. Lonser RR, Weil RJ, Wanebo JE, DeVroom HL, Oldfield EH: Surgical management of spinal cord hemangioblastomas in patients with von Hippel-Lindau disease. J Neurosurg 98:106-116, 2003

5. Martirosyan NL, Feuerstein JS, Theodore N, Cavalcanti DD, Spetzler RF, Preul MC: Blood supply and vascular reactivity of the spinal cord under normal and pathological conditions. J Neurosurg Spine 15:238-251, 2011

6. Mehta GU, Asthagiri AR, Bakhtian KD, Auh S, Oldfield EH, Lonser RR: Functional outcome after resection of spinal cord hemangioblastomas associated with von Hippel-Lindau disease. J Neurosurg Spine 12:233-242, 2010

7. Na JH, Kim HS, Eoh W, Kim JH, Kim JS, Kim ES: Spinal cord hemangioblastoma: diagnosis and clinical outcome after surgical treatment. J Korean Neurosurg Soc 42:436-440, 2007

8. Pan J, Ho AL, D'Astous M, Sussman ES, Thompson PA, Tayag AT, et al: Image-guided stereotactic radiosurgery for treatment of spinal hemangioblastoma. Neurosurg Focus 42(1):E12, 2017

9. Pluta RM, Iuliano B, DeVroom HL, Nguyen T, Oldfield EH: Comparison of anterior and posterior surgical approaches in the treatment of ventral spinal hemangioblastomas in patients with von Hippel-Lindau disease. J Neurosurg 98:117-124, 2003

10. Siller S, Szelényi A, Herlitz L, Tonn JC, Zausinger S: Spinal cord hemangioblastomas: significance of intraoperative neurophysiological monitoring for resection and long-term outcome. J Neurosurg Spine 26:483-493, 2017

11. Van Velthoven V, Reinacher PC, Klisch J, Neumann HPH, Gläsker S: Treatment of intramedullary hemangioblastomas, with special attention to von Hippel-Lindau disease. Neurosurgery 53:1306-1314, 2003

\section{Disclosures}

Professor Gläsker is supported by the Fund for Scientific Research-Flanders (FWO-V 1831017N). The FWO is a neutral organization without competing interests in the publication of this article.

\section{Author Contributions}

Conception and design: Van Der Veken. Acquisition of data: Van Der Veken. Analysis and interpretation of data: Van Der Veken. Drafting the article: Van Der Veken. Critically revising the article: Gläsker, Vougioukas. Reviewed submitted version of manuscript: Van Velthoven. Study supervision: Gläsker.

\section{Correspondence}

Jorn Van Der Veken: Universitair Ziekenhuis Brussel, Brussels, Belgium. jornvanderveken@gmail.com. 